\title{
Influencia de las condiciones de temperatura e iluminación en la rotura de latencia y germinación de los endemismos mediterráneos Scilla paui y Scilla ramburei (Liliaceae)
}

\author{
Influence of temperature and light conditions on dormancy break and germination \\ of the Mediterranean endemism of Scilla paui and Scilla ramburei (Liliaceae)
}

\author{
Alejandro Santiago ${ }^{\text {a,b }}$, José $\mathbf{M}^{\mathrm{a}}$ Herranz $^{\text {a,b }}$, Miguel Ángel Copete ${ }^{\mathrm{b} *}$, Raquel Herranz ${ }^{\mathrm{b}}$, Pablo Ferrandis ${ }^{\mathrm{b}}$ \\ a Jardín Botánico de Castilla-La Mancha, Avda. de la Mancha s/n, 02006, Albacete, España.

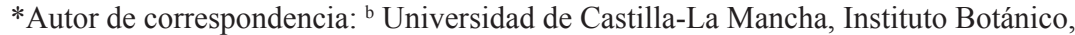 \\ Avda. de la Mancha s/n, 02006, Albacete, España, tel.: +34967599200, miguel.copete@uclm.es
}

\section{SUMMARY}

We studied the germination ecology of Scilla paui and Scilla ramburei to check the existence of underdeveloped embryos, as well as to verify the presence of any physiological or morphophysiological dormancy levels described for the family Liliaceae. Seeds of $S$. paui had fully developed embryos at seed dispersal and they were physiologically dormant. After eight weeks of cold (5 ${ }^{\circ} \mathrm{C}$ ) stratification followed by a four-week incubation at $15 / 4{ }^{\circ} \mathrm{C}$ in darkness, germination was $100 \%$. This record, along with the promotion of germination mediated by gibberellic acid, pointed out that seeds of $S$. paui had non-deep physiological dormancy. In $S$. ramburei seeds, the average embryo length at dispersal was $1.60 \mathrm{~mm}$; they had to grow to the critical length of $2.14 \mathrm{~mm}$ to be able to germinate. Embryo growth and radicle emergence occurred after seed exposure to a sequence of temperatures typical of the end summer-beginning autumn period. Emerged radicles required two months of exposition to winter cold $\left(5^{\circ} \mathrm{C}\right)$ for seedling emergence in February and March. Seeds of S. ramburei had deep simple epicotyl morphophysiological dormancy. The differences detected in the germination ecology support the segregation of both taxa as different species, and suggest the convenience of taking into account physiological traits in systematic studies.

Key words: underdeveloped embryos, morphophysiological dormancy, physiological dormancy.

\section{RESUMEN}

Se analizó la ecología germinativa de Scilla paui y Scilla ramburei para comprobar la posible existencia de embriones subdesarrollados, así como para verificar si poseían alguno de los tipos de latencia conocidos para Liliaceae. Las semillas de $S$. paui presentaron embriones perfectamente desarrollados al dispersar sus semillas y latencia fisiológica. Tras ocho semanas de estratificación fría (5 ${ }^{\circ} \mathrm{C}$ ) y posterior incubación durante cuatro semanas a $15 / 4{ }^{\circ} \mathrm{C}$ en oscuridad, se alcanzó el $100 \%$ de semillas germinadas. Este hecho, unido al efecto estimulador del ácido giberélico, indicó que las semillas de $S$. paui poseían latencia fisiológica no profunda. En $S$. ramburei el tamaño medio del embrión al dispersar fue $1,60 \mathrm{~mm}$, y para poder germinar tuvo que crecer hasta el tamaño crítico de 2,14 $\mathrm{mm}$. El crecimiento del embrión y posterior emergencia de las radículas se produjo tras la exposición de las semillas a una secuencia de temperaturas propias de finales de verano y principios de otoño. Tras la emergencia de la radícula, a finales de noviembre, se requirieron dos meses de exposición al frío invernal $\left(5^{\circ} \mathrm{C}\right)$ para la emergencia de las plántulas en febrero y marzo. Las semillas de $S$. ramburei mostraron latencia morfofisiológica simple y profunda del epicotilo. Las diferencias en ecología germinativa apoyan el mantenimiento de ambos taxones como especies diferentes, así como la conveniencia de tener en cuenta los caracteres fisiológicos en los estudios de sistemática.

Palabras clave: embriones subdesarrollados, latencia morfofisiológica, latencia fisiológica.

\section{INTRODUCCIÓN}

En muchas familias de plantas, como Liliaceae, existen especies con semillas que en el momento de la dispersión poseen un embrión subdesarrollado (pequeño y que tiene que crecer dentro de la semilla antes de que la radícula pueda emerger), unido a un mecanismo fisiológico adicional que dificulta el crecimiento del embrión y la ger- minación. Estas semillas tienen latencia morfofisiológica (Baskin y Baskin 2004). Se han descrito nueve niveles de ella en función de: a) temperaturas requeridas para la rotura de latencia, b) temperaturas requeridas para el crecimiento del embrión, y c) capacidad del ácido giberélico $\left(\mathrm{GA}_{3}\right)$ para sustituir una estratificación fría y/o cálida en la rotura de latencia (Baskin et al. 2008, Phartyal et al. 2009). La latencia morfofisiológica está considerada como 
la más difícil de superar (Baskin y Baskin 1998), por lo que los estudios sobre la misma son relativamente escasos en comparación con otras clases de latencia, especialmente en el ámbito circunmediterráneo (Herranz et al. 2010).

Dentro de la familia Liliaceae, se ha comprobado la existencia de latencia fisiológica (Baskin y Baskin 1998), así como varios niveles de latencia morfofisiológica: simple y profunda en Cardiocrinum cordatum (Thunb.) Makino (Kondo et al. 2006); simple y profunda del epicotilo en Gagea lutea (L) Ker Gawl. (Kondo et al. 2004); simple, profunda y doble en Convallaria majalis L. y Polygonatum biflorum (Walter) Elliott (Baskin y Baskin 1998); compleja y no profunda en Erythronium albidum Nutt. (Baskin y Baskin 1985a); y compleja profunda en Erythronium grandiflorum Pursh. (Baskin et al. 1995).

Los estudios de germinación en el género Scilla son escasos. Los realizados en S. scilloides (Lindl) Druce (Washitani y Masuda 1990) y S. bifolia L. (Vandelook y Van Assche 2008) pusieron de manifiesto la existencia de latencia morfofisiológica y los llevados a cabo en S. autumnalis L. (Marques y Draper 2012) la ausencia de latencia.

En este trabajo se estudia la ecología germinativa de dos especies mediterráneas geófitas bulbosas del género Scilla con distribución geográfica muy restringida. Scilla paui Lacaita es un endemismo ibérico de las cordilleras subbéticas (Alcaraz, Segura y Cazorla) del sur de España que crece entre 900-1.700 m de altitud (Navarro 2009), formando parte de hábitats de protección especial como pinares de Pinus nigra Arnold subsp. salzmannii (Dunal) Franco y comunidades orófilas dolomitícolas subbéticas (Herranz et al. 2011). Scilla ramburei Boiss. es un endemismo ibero-norteafricano que, en la península Ibérica, solo se halla en algunas sierras meridionales (Morena, Cazorla) entre 600 y $1.000 \mathrm{~m}$ de altitud (Navarro 2009), siendo un elemento destacado del hábitat de protección especial juncales higroturbosos silicícolas (Herranz et al. 2011). Debido a la escasez de sus poblaciones y a su reducido número de efectivos, ambas especies han sido protegidas en la Comunidad de Castilla-La Mancha (España), ubicada en el interior peninsular (DOCM 1998). La consecución de un estado de conservación favorable para ambas especies, así como para sus hábitats, puede requerir la necesidad de efectuar reforzamientos poblacionales, utilizando individuos producidos a partir de semillas para maximizar la diversidad genética, siendo para ello fundamental el conocimiento de su ecología germinativa (Cerabolini et al. 2004).

Estudios previos sobre estas especies llevados a cabo por este equipo de investigación, en el ámbito del conocimiento de la ecología germinativa de diferentes monocotiledóneas ibéricas (Copete et al. 2011), apuntaban a la existencia de embriones perfectamente desarrollados en $S$. paui y subdesarrollados en $S$. ramburei en el momento de la dispersión de las semillas y a la ausencia de germinación sin estratificación previa. En consecuencia, en este estudio se plantea la hipótesis de una latencia fisiológica en las semillas de $S$. paui y de alguno de los niveles de latencia morfofisiológica citados para Liliaceae en $S$. ramburei. Para ello, se analizan las condiciones de temperatura e iluminación precisas para la rotura de latencia y germinación en ambas especies.

Los objetivos concretos son los siguientes:

1. Comprobar si presentan embriones subdesarrollados y evaluar los efectos de la temperatura para la rotura de latencia y crecimiento del embrión.

2. Analizar la influencia de la duración de la estratificación cálida y/o fría, de las condiciones de iluminación y del ácido giberélico $\left(\mathrm{GA}_{3}\right)$ en la capacidad germinativa de las semillas.

3. Determinar si las temperaturas de finales de verano pueden inducir latencia en semillas no latentes de S. paui.

4. Evaluar el efecto de las temperaturas características de estratificación fría $\left(5^{\circ} \mathrm{C}\right)$ sobre la emergencia de plántulas en semillas de $S$. ramburei con radículas emergidas.

5. Analizar la fenología de crecimiento del embrión, de rotura de latencia, y de emergencia de radículas y plántulas en condiciones semejantes a las naturales.

6. Contribuir al esclarecimiento taxonómico de ambas especies en función de sus diferencias en ecología germinativa.

\section{MÉTODOS}

Fuente de las semillas. Las semillas de S. paui fueron recolectadas el 17 de junio de 2009 a una altitud de 1.200 $\mathrm{m}$ en el paraje de "La Merera", dentro del término municipal de Peñascosa, Sierra de Alcaraz, en la provincia de Albacete (30SWH5436), en claros de pinar de Pinus nigra subsp. salzmannii, con Cytisus scoparius (L.) Link subsp. reverchonii (Degen et Hervier) Rivas Goday et Rivas Mart., Echinospartum boissieri (Spach) Rothm. y Thymus granatensis Boiss., con sustrato de fondo calizo y suelo arcilloso. Se recolectaron aproximadamente 8.000 semillas provenientes de unas 200 cápsulas maduras con inicio de apertura apical. Las semillas de Scilla ramburei utilizadas en los ensayos provenían de una población localizada a $820 \mathrm{~m}$ de altitud a orillas del arroyo del Enebrillo en Sierra Madrona, en el término municipal de Fuencaliente, Ciudad Real (30SUH8356) y fueron recolectadas el 8 de julio de 2009, en el sotobosque de una aliseda (Alnus glutinosa (L.) Gaertn.) con Osmunda regalis L., Hypericum androsaemum L. y Ornithogalum pyrenaicum L., sobre sustrato de cuarcitas y pizarras. Se recolectaron unas 12.000 semillas procedentes de unas 350 cápsulas de color amarillo, indicativo de su madurez. Tras su recolección, las semillas se dejaron secar en el laboratorio durante dos semanas y, a continuación, se conservaron en sobres de papel aproximadamente a $20-22{ }^{\circ} \mathrm{C}$ y un $60 \%$ de humedad relativa, hasta el inicio de los ensayos, en septiembre de 2009. 
Ensayos de laboratorio. Los experimentos fueron realizados bajo condiciones de temperatura e iluminación controladas en cámaras de germinación (Ibercex, modelo F-4, Madrid) equipadas con control digital. Las cámaras fueron programadas con $12 \mathrm{~h}$ diarias de fotoperiodo y las semillas, colocadas en placas de Petri sobre dos láminas de papel de filtro humedecido con agua destilada, fueron expuestas a este fotoperiodo (tratamiento en luz), o bien permanecieron bajo condiciones de oscuridad lograda envolviendo las placas Petri en dos láminas de papel de aluminio (tratamiento en oscuridad). Los experimentos se llevaron a cabo tanto a la temperatura constante de $5^{\circ} \mathrm{C}$ como a un régimen de $12 / 12 \mathrm{~h}$ diarias con temperaturas fluctuantes de $15 / 4,20 / 7,25 / 10$ y $28 / 14{ }^{\circ} \mathrm{C}$. En este régimen fluctuante, la temperatura más alta coincidió con la fase de luz y la más baja con la fase de oscuridad. En cada ensayo se utilizaron cuatro réplicas de 25 semillas cada una. Las temperaturas utilizadas en los ensayos de germinación trataron de simular las condiciones climáticas existentes en ambientes mediterráneos de 800-1.200 m de altitud a lo largo del año: la temperatura constante de $5^{\circ} \mathrm{C}$ se aproxima a la temperatura media durante los meses invernales (diciembre, enero, febrero), $15 / 4{ }^{\circ} \mathrm{C}$ a las de noviembre y marzo, $20 / 7{ }^{\circ} \mathrm{C}$ a las de octubre y abril, $25 / 10^{\circ} \mathrm{C}$ a las de septiembre y mayo, y $28 / 14{ }^{\circ} \mathrm{C}$ a las de junio, julio y agosto.

Comprobación de la existencia de embriones subdesarrollados y efecto de la temperatura en el crecimiento del embrión. El 30 de junio de 2009 se depositaron 25 semillas de S. paui en una placa Petri de $9 \mathrm{~cm}$ de diámetro sobre dos capas de papel de filtro humedecido con agua destilada. Después de $24 \mathrm{~h}$ a temperatura ambiente de laboratorio $\left(22^{\circ} \mathrm{C}\right)$, las semillas fueron seccionadas con un bisturí para obtener los embriones y medir su tamaño mediante un microscopio equipado con micrómetro. El tamaño medio de los embriones (E) en semillas recién dispersadas fue de
$1,59 \mathrm{~mm}$, frente a un tamaño medio de las semillas (S) de $1,98 \mathrm{~mm}$, siendo el ratio $\mathrm{E}: \mathrm{S}=0,80$. Cuando este ratio es $\geq 0,60$ indica ausencia de embriones subdesarrollados (Baskin y Baskin 1998). En ensayos posteriores se pudo confirmar que el tamaño medio del embrión en semillas recién germinadas era de $1,59 \mathrm{~mm}$.

El 20 de julio de 2009 se midió el tamaño de los embriones en semillas dispersadas de $S$. ramburei, procediendo de la forma indicada anteriormente para $S$. paui. El tamaño medio del embrión en semillas recién dispersadas fue de $1,60 \mathrm{~mm}$ y el tamaño medio de las semillas $2,68 \mathrm{~mm}$, siendo el ratio $\mathrm{E}: \mathrm{S}<0,60(=0,56)$, lo que parecía indicar la presencia de embriones subdesarrollados, como se confirmó tras la medición de embriones en semillas a punto de emerger la radícula. Para promover la germinación se estratificó un lote de 200 semillas a $28 / 14^{\circ} \mathrm{C}$ en luz durante ocho semanas y posteriormente se incubaron a $15 / 4{ }^{\circ} \mathrm{C}$ en oscuridad durante cuatro semanas. En 40 de estas semillas con la testa rota, pero sin llegar a producirse la emergencia de la radícula, se midió el embrión y se calculó el valor medio, obteniéndose de esta forma el tamaño crítico del embrión (Vandelook et al. 2009, Phartyal et al. 2009). El tamaño crítico resultó ser 2,14 mm, con un rango de oscilación entre 1,9-2,6 mm. El valor de 1,9 mm es el tamaño mínimo de embrión a partir del cual se puede producir la germinación.

Para evaluar la influencia de la temperatura en el crecimiento del embrión de $S$. ramburei se efectuaron seis ensayos de estratificación del tipo "move-along" (Baskin y Baskin 2004), siguiendo una secuencia de temperaturas de verano $\rightarrow$ otoño $\rightarrow$ invierno $\rightarrow$ primavera, con una duración del periodo de estratificación entre 20-28 semanas, bajo condiciones de fotoperiodo. En los ensayos de estratificación adicionales, las semillas fueron expuestas a la temperatura fluctuante de $28 / 14{ }^{\circ} \mathrm{C}$ durante 24 semanas en uno (E7) y a la temperatura constante de $5{ }^{\circ} \mathrm{C}$ durante 24 semanas en el otro (E8) (cuadro 1).

Cuadro 1. Secuencia de temperaturas fluctuantes decrecientes (move-along), así como temperaturas fijas, en ensayos de estratificación para $S$. ramburei. Entre paréntesis se indica la duración del periodo de estratificación en semanas.

Sequence of decreasing fluctuating temperatures (move-along), and stable temperatures, in seed stratification treatments for $S$. ramburei. Number between brackets shows length of stratification in weeks.

\begin{tabular}{|c|c|}
\hline & Ensayos de estratificación \\
\hline E1 & $28 / 14^{\circ} \mathrm{C}(4 \mathrm{~s}) \rightarrow 25 / 10^{\circ} \mathrm{C}(4 \mathrm{~s}) \rightarrow 20 / 7^{\circ} \mathrm{C}(4 \mathrm{~s}) \rightarrow 15 / 4{ }^{\circ} \mathrm{C}(4 \mathrm{~s}) \rightarrow 5^{\circ} \mathrm{C}(8 \mathrm{~s})$ \\
\hline E2 & $28 / 14^{\circ} \mathrm{C}(4 \mathrm{~s}) \rightarrow 25 / 10^{\circ} \mathrm{C}(4 \mathrm{~s}) \rightarrow 20 / 7^{\circ} \mathrm{C}(4 \mathrm{~s}) \rightarrow 15 / 4^{\circ} \mathrm{C}(4 \mathrm{~s}) \rightarrow 5^{\circ} \mathrm{C}(4 \mathrm{~s}) \rightarrow 15 / 4^{\circ} \mathrm{C}(4 \mathrm{~s})$ \\
\hline E3 & $28 / 14^{\circ} \mathrm{C}(4 \mathrm{~s}) \rightarrow 25 / 10^{\circ} \mathrm{C}(8 \mathrm{~s}) \rightarrow 20 / 7^{\circ} \mathrm{C}(4 \mathrm{~s}) \rightarrow 15 / 4^{\circ} \mathrm{C}(4 \mathrm{~s}) \rightarrow 5^{\circ} \mathrm{C}(4 \mathrm{~s}) \rightarrow 15 / 4{ }^{\circ} \mathrm{C}(4 \mathrm{~s})$ \\
\hline E4 & $28 / 14^{\circ} \mathrm{C}(12 \mathrm{~s}) \rightarrow 20 / 7^{\circ} \mathrm{C}(4 \mathrm{~s}) \rightarrow 15 / 4^{\circ} \mathrm{C}(4 \mathrm{~s}) \rightarrow 5^{\circ} \mathrm{C}(4 \mathrm{~s}) \rightarrow 15 / 4^{\circ} \mathrm{C}(4 \mathrm{~s})$ \\
\hline E5 & $25 / 10^{\circ} \mathrm{C}(4 \mathrm{~s}) \rightarrow 20 / 7^{\circ} \mathrm{C}(4 \mathrm{~s}) \rightarrow 15 / 4^{\circ} \mathrm{C}(4 \mathrm{~s}) \rightarrow 5^{\circ} \mathrm{C}(8 \mathrm{~s})$ \\
\hline E6 & $25 / 10^{\circ} \mathrm{C}(4 \mathrm{~s}) \rightarrow 20 / 7^{\circ} \mathrm{C}(4 \mathrm{~s}) \rightarrow 15 / 4^{\circ} \mathrm{C}(4 \mathrm{~s}) \rightarrow 5^{\circ} \mathrm{C}(4 \mathrm{~s}) \quad \rightarrow 15 / 4^{\circ} \mathrm{C}(4 \mathrm{~s})$ \\
\hline E7 & $28 / 14{ }^{\circ} \mathrm{C}(24 \mathrm{~s})$ \\
\hline E8 & $5^{\circ} \mathrm{C}(24 s)$ \\
\hline
\end{tabular}


Para cada uno de los ensayos de estratificación del tipo "move-along" se utilizaron siete placas Petri con 25 semillas cada una que fueron movidas de una temperatura a otra siguiendo la secuencia que se indica a continuación. En cada ensayo se midió una muestra de 25 embriones cada cuatro semanas.

Para cada uno de los ensayos a $5{ }^{\circ} \mathrm{C}$ o $28 / 14{ }^{\circ} \mathrm{C}$ durante todo el periodo de estratificación, solo se utilizó una placa Petri con 50 semillas, ya que solo se efectuó una medición de 25 embriones al final del ensayo.

Influencia de la temperatura de estratificación y condiciones de iluminación sobre la capacidad germinativa. En $S$. paui, los ensayos preliminares efectuados durante el mes de julio de 2009 a las temperaturas de 5, 15/4, 20/7 y 25/10 ${ }^{\circ} \mathrm{C}$, tanto en luz como en oscuridad, arrojaron una germinación nula. En consecuencia, el 1 de septiembre de 2009 se iniciaron los ensayos de estratificación. Para ello se prepararon seis lotes de semillas, con 800 semillas cada uno, dispuestas en placas Petri. Dos lotes (uno en condiciones de luz y otro en oscuridad) fueron expuestos a cada una de las siguientes secuencias de temperatura:

$$
\begin{aligned}
& \text { E1: } 5{ }^{\circ} \mathrm{C}(8 \mathrm{~s}) \\
& \text { E2: } 15 / 4{ }^{\circ} \mathrm{C}(4 \mathrm{~s}) \rightarrow 5^{\circ} \mathrm{C}(4 \mathrm{~s}) \\
& \text { E3: } 28 / 14^{\circ} \mathrm{C}(8 \mathrm{~s})
\end{aligned}
$$

Después de estos tratamientos de estratificación, las semillas se incubaron durante cuatro semanas a 5, 15/4, $20 / 7$ y $25 / 10{ }^{\circ} \mathrm{C}$ en luz y oscuridad. Además, se efectuó un ensayo control, con incubación durante 12 semanas de semillas no estratificadas previamente a las temperaturas citadas en el párrafo anterior.

Los altos porcentajes de germinación obtenidos tras una estratificación fría $\left(5^{\circ} \mathrm{C}\right)$ durante ocho semanas, llevaron a probar los efectos de periodos de estratificación más cortos. Por ello, el 1 de diciembre de 2009, se prepararon 10 placas Petri con 200 semillas/placa que fueron estratificadas a $5{ }^{\circ} \mathrm{C}$ (5 placas en luz y 5 en oscuridad) durante $1,2,3,4$ y 5 semanas. Tras cada uno de estos periodos, las semillas fueron incubadas durante cuatro semanas a $15 / 4^{\circ} \mathrm{C}$ en luz y oscuridad.

En $S$. ramburei, los ensayos preliminares efectuados durante agosto de 2009 en las mismas condiciones de temperatura e iluminación que para $S$. paui dieron germinación nula. Este hecho, unido a la presencia de embriones subdesarrollados, llevó a planificar ensayos de estratificación de larga duración. El 1 de septiembre se iniciaron los ensayos de estratificación en condiciones de fotoperiodo indicados en el cuadro 1. Para cada ensayo se dispuso una placa Petri con 800 semillas. Finalizado el periodo de estratificación, se eliminaron las semillas ya germinadas y se incubaron las restantes a $5,15 / 4$ y $20 / 7^{\circ} \mathrm{C}$, tanto en luz como en oscuridad durante cuatro semanas. También se efectuó un ensayo control, incubando durante 28 semanas a $5,15 / 4$ y $20 / 7^{\circ} \mathrm{C}$, tanto en luz como en oscuridad, semillas no sometidas a estratificación previa.
Efectos del $\mathrm{GA}_{3}$ sobre la capacidad germinativa. Este ensayo se realizó el 1 de septiembre de 2009. Para cada especie, se utilizaron 24 réplicas de 25 semillas cada una, que se dispusieron en placas Petri de $9 \mathrm{~cm}$ de diámetro sobre dos capas de papel de filtro humedecido con una solución de $1.000 \mathrm{mg} \mathrm{L}^{-1}$ de $\mathrm{GA}_{3}$. Doce réplicas fueron colocadas a $20 / 7^{\circ} \mathrm{C}$ en luz y otras 12 a igual temperatura en oscuridad. Después de 4,8 y 12 semanas, se tomaron cuatro placas Petri de cada condición de iluminación y se contabilizó el número de semillas germinadas. A efectos comparativos, se realizó un ensayo control exactamente igual al que se acaba de describir, pero colocando las semillas sobre papel de filtro humedecido únicamente con agua destilada.

Inducción de latencia por altas temperaturas en Scilla paui. Es conocido que, en algunas especies que requieren estratificación fría para superar el componente de latencia fisiológica, la exposición de semillas no latentes a las temperaturas de finales de verano $\left(25 / 10^{\circ} \mathrm{C}\right)$ puede inducir de nuevo latencia (Herranz et al. 2010). Para verificar si esto sucedía en $S$. paui se realizó el siguiente experimento. El 1 de diciembre de 2009 se prepararon cuatro lotes con 200 semillas cada uno, que fueron sometidas a los siguientes tratamientos de estratificación con luz:

$$
\begin{aligned}
& \text { E1: } 5^{\circ} \mathrm{C}(8 \mathrm{~s}) \text { (control) } \\
& \mathrm{E} 2: 5^{\circ} \mathrm{C}(8 \mathrm{~s}) \rightarrow 25 / 10^{\circ} \mathrm{C}(4 \mathrm{~s}) \\
& \text { E3: } 5^{\circ} \mathrm{C}(8 \mathrm{~s}) \rightarrow 25 / 10{ }^{\circ} \mathrm{C}(4 \mathrm{~s}) \rightarrow 5^{\circ} \mathrm{C}(4 \mathrm{~s}) \\
& \text { E4: } 5^{\circ} \mathrm{C}(8 \mathrm{~s}) \rightarrow 25 / 10^{\circ} \mathrm{C}(4 \mathrm{~s}) \rightarrow 5^{\circ} \mathrm{C}(8 \mathrm{~s})
\end{aligned}
$$

Al final de cada tratamiento de estratificación las semillas se incubaron durante cuatro semanas a $15 / 4{ }^{\circ} \mathrm{C}$ en luz y oscuridad.

Efecto de la estratificación fría sobre la emergencia de plántulas en semillas de S. ramburei con radiculas emergidas. A lo largo de los ensayos de estratificación del tipo "move-along" iniciados el 1 de septiembre de 2009 para analizar el crecimiento del embrión con diferentes secuencias de temperaturas, se pudo comprobar que, en las semillas germinadas, emergía la radícula, pero no el cotiledón, lo que indicaba una posible latencia del epicotilo. Para verificar esta posible latencia, el 15 de marzo de 2010 se prepararon tres lotes de 75 semillas germinadas (radículas emergidas de 2-3 mm), que fueron expuestas a estratificación fría durante 0,4 y 8 semanas. Tras cada periodo de estratificación un lote de 75 semillas (dividido en tres réplicas de 25) fue incubado durante 60 días a $20 / 7{ }^{\circ} \mathrm{C}$ en luz, registrando el porcentaje de emergencia de cotiledones $(\geq 1 \mathrm{~mm})$.

Ensayos de campo en condiciones seminaturales. El objetivo de estos ensayos fue determinar los cambios en el estado de latencia, así como el momento de los principales eventos en el tránsito semilla-plántula, cuando las semillas son expuestas a los ciclos estacionales de temperatura 
imitando las condiciones naturales. Estos ensayos se realizaron en un umbráculo situado en el campo de prácticas de la Escuela Técnica Superior de Ingenieros Agrónomos y de Montes de Albacete. La temperatura fue registrada a lo largo del ensayo por una estación meteorológica. El sustrato de crecimiento en bandejas y macetas donde fueron sembradas las semillas estaba constituido por una mezcla de turba esterilizada y arena (2:1) y se regaron hasta la capacidad de campo una vez por semana durante todo el año, excepto en los meses de julio y agosto (dos veces al mes) para simular la sequía estival mediterránea. Asimismo, durante los periodos invernales en los que bandejas y macetas estaban heladas se interrumpió el riego.

Fenología del crecimiento del embrión, de rotura de latencia, y de emergencia de radículas y plántulas. El 1 de septiembre de 2009 se inició este ensayo con 12 lotes de 100 semillas para cada especie. Las semillas se mezclaron con arena y se guardaron en el interior de bolsas de nylon. Las 12 bolsas de cada especie se enterraron a $5 \mathrm{~cm}$ de profundidad en una maceta de $22 \mathrm{~cm}$ de diámetro por $18 \mathrm{~cm}$ de profundidad, y se colocaron en el umbráculo anteriormente descrito.

Las bolsas se desenterraron con periodicidad mensual a partir del mes siguiente al del inicio de cada ensayo, determinando el porcentaje de semillas con radículas emergidas y, en el caso de $S$. ramburei, se utilizó una muestra de 25 semillas para medir la evolución del crecimiento del embrión. Las semillas recuperadas en cada rescate, excluyendo las que tenían radículas emergidas y las 25 utilizadas para medir el crecimiento del embrión en S. ramburei, se incubaron a $15 / 4{ }^{\circ} \mathrm{C}$ en oscuridad durante 30 días. Con el resultado del periodo de incubación se pudieron calcular los siguientes porcentajes: a) semillas germinadas en la bolsa (con radículas emergidas), b) semillas viables latentes (con embrión sano y no germinadas durante la incubación), c) semillas viables no latentes (germinadas durante la incubación), d) semillas inviables (las recuperadas en mal estado, así como las que presentaban embriones con mal aspecto tras la incubación).

Asimismo, el 1 de septiembre de 2009 se sembraron tres réplicas de 100 semillas cada una para cada especie en bandejas de plástico $(40 \times 30 \times 5 \mathrm{~cm})$ provistas de agujeros de drenaje. Las bandejas se rellenaron con el sustrato descrito anteriormente y la profundidad de siembra fue de 3-5 $\mathrm{mm}$. Con periodicidad semanal se registró la emergencia de plántulas durante 18 meses, retirándose las emergidas.

Análisis estadístico. Para cada ensayo, se calcularon los porcentajes de germinación acumulados sobre semillas viables, considerando viables las semillas germinadas y las no germinadas con embriones blanquecinos y turgentes. Se calculó la media y el error estándar tanto para los datos de germinación $(\mathrm{n}=4)$ como para los datos de crecimiento de embrión $(\mathrm{n}=25)$. Mediante ANDEVA multifactorial se analizó el efecto del fotoperiodo, de la tempe- ratura durante la estratificación y de la temperatura durante la incubación, tanto sobre el crecimiento del embrión, como sobre el porcentaje final de germinación. En todos los casos, los factores responsables de los efectos principales fueron detectados mediante una comparación múltiple realizada con la prueba de Tukey (al nivel de significación de 0,05 ). Como paso previo al análisis, se comprobaron las condiciones de normalidad (prueba de Cochran) y homocedasticidad (prueba de David). Los porcentajes de germinación se transformaron para su análisis estadístico en los valores de la raíz cuadrada de su arcoseno, para ajustar su distribución a la normal. En las representaciones gráficas que incluyen dichos porcentajes estos son representados sin transformar.

\section{RESULTADOS}

Efecto de la temperatura en el crecimiento de los embriones de S. ramburei. En todos los ensayos de estratificación en los que las semillas fueron expuestas durante largos periodos a secuencias de temperaturas cálidas (características de los meses de verano), y/o moderadamente cálidas (meses de otoño), los embriones crecieron hasta alcanzar los $2 \mathrm{~mm}$ de longitud. Asimismo, tras 24 semanas a la temperatura fluctuante de $28 / 14^{\circ} \mathrm{C}$, los embriones crecieron hasta $1,94 \mathrm{~mm}$. Sin embargo, tras 24 semanas a $5{ }^{\circ} \mathrm{C}$, los embriones sólo crecieron hasta 1,67 mm (cuadro 2).

Un $25 \%$ de las semillas del ensayo de estratificación E3 llegaron a germinar y un $30 \%$ superaron el tamaño mínimo de $1,9 \mathrm{~mm}$, a partir del cual la germinación es posible (cuadro 2).

Influencia de la temperatura de estratificación y condiciones de iluminación sobre la capacidad germinativa. Durante la prueba de estratificación iniciada en septiembre de 2009 se pudo observar que las condiciones de incubación en las que se produjo mayor germinación de las semillas de $S$. paui fueron de cuatro semanas a las temperaturas fluctuantes de $15 / 4{ }^{\circ} \mathrm{C}$, tanto en luz como en oscuridad. Así, en las semillas que habían sido sometidas a los tratamientos de estratificación fría E1 y E2, en ambas condiciones de iluminación, se superaba el $95 \%$ de germinación. Tras el tratamiento de estratificación cálida la germinación fue nula (cuadro 3).

Al final del ensayo control (12 semanas) algunos de los porcentajes de germinación alcanzados fueron: $55 \%$ a $5{ }^{\circ} \mathrm{C}$ luz y $95 \%$ a $5{ }^{\circ} \mathrm{C}$ oscuridad; $92 \%$ a $15 / 4{ }^{\circ} \mathrm{C}$ luz y $99 \%$ a $15 / 4{ }^{\circ} \mathrm{C}$ oscuridad. A $20 / 7{ }^{\circ} \mathrm{C}$ y $25 / 10^{\circ} \mathrm{C}$ la germinación fue nula.

En el ensayo iniciado el 1 de diciembre de 2009 con periodos de estratificación más cortos, los mayores porcentajes de germinación se obtuvieron con una estratificación fría a $5{ }^{\circ} \mathrm{C}$ en oscuridad y posterior incubación de cuatro semanas a $15 / 4{ }^{\circ} \mathrm{C}$ también en oscuridad, siendo del $0 \%, 16 \%, 30 \%, 72 \%$ y $100 \%$ después de $1,2,3,4$ y 5 semanas de estratificación, respectivamente (figura 1). 
Cuadro 2. Crecimiento de embrión en semillas de S. ramburei sometidas a los tratamientos de estratificación indicados en el cuadro 1. El primer número que aparece entre paréntesis muestra el porcentaje de semillas germinadas, y el segundo el porcentaje de semillas con tamaño de embrión $\geq 1,9 \mathrm{~mm}$, valor mínimo a partir del cual es posible la germinación.

Embryo growth in S. ramburei seeds submitted to stratification treatments shown in Table 1. The first number between brackets shows the percentage of seeds germinated, and the second one indicates the percentage of seeds with embryo length $\geq 1.9 \mathrm{~mm}$, minimum value from which germination is possible.

\begin{tabular}{|c|c|c|c|c|c|c|c|}
\hline \multirow{2}{*}{$\begin{array}{l}\text { Tratamiento de } \\
\text { estratificación }\end{array}$} & \multirow{2}{*}{ Característica } & \multicolumn{6}{|c|}{ Temperatura de estratificación en luz } \\
\hline & & $28 / 14^{\circ} \mathrm{C}$ & $25 / 10^{\circ} \mathrm{C}$ & $20 / 7^{\circ} \mathrm{C}$ & $15 / 4{ }^{\circ} \mathrm{C}$ & $5^{\circ} \mathrm{C}$ & $15 / 4^{\circ} \mathrm{C}$ \\
\hline \multirow{3}{*}{ E1 } & $\mathrm{N}^{\mathrm{o}}$ semanas & 4 & 4 & 4 & 4 & 8 & \\
\hline & Embrión (mm) & 1,66 & 1,66 & 1,78 & 1,97 & 2,07 & \\
\hline & Germinación (\%) & $(0 ; 4)$ & $(0 ; 6)$ & $(0 ; 12)$ & $(6 ; 8)$ & $(7 ; 8)$ & \\
\hline \multirow{3}{*}{ E2 } & $\mathrm{N}^{\mathrm{o}}$ semanas & 4 & 4 & 4 & 4 & 4 & 4 \\
\hline & Embrión (mm) & 1,66 & 1,66 & 1,78 & 1,97 & 2,01 & 2,06 \\
\hline & Germinación (\%) & $(0 ; 4)$ & $(0 ; 6)$ & $(0 ; 12)$ & $(5 ; 8)$ & $(6 ; 8)$ & $(6 ; 8)$ \\
\hline \multirow{3}{*}{ E3 } & $\mathrm{N}^{\circ}$ semanas & 4 & 8 & 4 & 4 & 4 & 4 \\
\hline & Embrión (mm) & 1,66 & 1,68 & 1,81 & 1,98 & 2,06 & 2,07 \\
\hline & Germinación (\%) & $(0 ; 4)$ & $(0 ; 11)$ & $(0 ; 12)$ & $(8 ; 19)$ & $(4 ; 18)$ & $(25 ; 30)$ \\
\hline \multirow{3}{*}{ E4 } & $\mathrm{N}^{\mathrm{o}}$ semanas & 12 & & 4 & 4 & 4 & 4 \\
\hline & Embrión (mm) & 1,82 & & 1,85 & 2,0 & 2,06 & 2,06 \\
\hline & Germinación (\%) & $(0 ; 12)$ & & $(0 ; 12)$ & $(4 ; 20)$ & $(4 ; 16)$ & $(18 ; 20)$ \\
\hline \multirow{3}{*}{ E5 } & $\mathrm{N}^{\mathrm{o}}$ semanas & & 4 & 4 & 4 & 8 & \\
\hline & Embrión (mm) & & 1,72 & 1,78 & 1,81 & 2,02 & \\
\hline & Germinación (\%) & & $(0 ; 4)$ & $(0 ; 12)$ & $(0 ; 14)$ & $(0 ; 20)$ & \\
\hline \multirow{3}{*}{ E6 } & $\mathrm{N}^{\mathrm{o}}$ semanas & & 4 & 4 & 4 & 4 & 4 \\
\hline & Embrión (mm) & & 1,72 & 1,78 & 1,81 & 2,06 & 2,06 \\
\hline & Germinación (\%) & & $(0 ; 4)$ & $(0 ; 12)$ & $(0 ; 4)$ & $(8 ; 10)$ & $(8 ; 16)$ \\
\hline \multirow{3}{*}{ E7 } & $\mathrm{N}^{\mathrm{o}}$ semanas & 24 & & & & & \\
\hline & Embrión (mm) & 1,94 & & & & & \\
\hline & Germinación (\%) & $(0 ; 16)$ & & & & & \\
\hline \multirow{3}{*}{ E8 } & $\mathrm{N}^{\mathrm{o}}$ semanas & & & & & 24 & \\
\hline & Embrión (mm) & & & & & 1,67 & \\
\hline & Germinación (\%) & & & & & $(0 ; 0)$ & \\
\hline
\end{tabular}

Cuadro 3. Porcentaje de germinación (media $\pm \mathrm{SE}, \mathrm{n}=4$ ) en semillas de $S$. paui sometidas a los siguientes tratamientos de estratificación tanto en luz (L) como en oscuridad (O): E1: $5{ }^{\circ} \mathrm{C}\left(8\right.$ semanas); E2: $15 / 4{ }^{\circ} \mathrm{C}(4$ semanas $) \rightarrow 5{ }^{\circ} \mathrm{C}(4$ semanas $)$; E3: $28 / 14{ }^{\circ} \mathrm{C}(8$ semanas). Los tratamientos de estratificación fueron seguidos de cuatro semanas de incubación a $5{ }^{\circ} \mathrm{C}, 15 / 4{ }^{\circ} \mathrm{C}, 20 / 7{ }^{\circ} \mathrm{C}$ y $25 / 10{ }^{\circ} \mathrm{C}$ tanto en luz como en oscuridad. Las letras mayúsculas en columnas y minúsculas en filas denotan diferencias significativas $(P<0,05)$.

Germination percentage (mean $\pm \mathrm{SE}, \mathrm{n}=4)$ in $S$. paui seeds submitted to the following stratification treatments, both in light (L) and darkness (O): E1: $5^{\circ} \mathrm{C}$ (8 weeks); E2: $15 / 4{ }^{\circ} \mathrm{C}$ (4 weeks) $\rightarrow 5^{\circ} \mathrm{C}\left(4\right.$ weeks); E3: $28 / 14{ }^{\circ} \mathrm{C}$ ( 8 weeks). Stratification treatments were followed by four weeks of incubation at $5^{\circ} \mathrm{C}, 15 / 4^{\circ} \mathrm{C}, 20 / 7^{\circ} \mathrm{C}$ and $25 / 10^{\circ} \mathrm{C}$, both in light and darkness. Different uppercase letters in columns and lowercase letters in rows denote significant differences $(P<0.05)$.

\begin{tabular}{|c|c|c|c|c|c|c|c|c|c|}
\hline \multirow{3}{*}{$\begin{array}{l}\text { Tratamiento } \\
\text { Estratificación }\end{array}$} & & \multicolumn{8}{|c|}{ Temperaturas de incubación (cuatro semanas) } \\
\hline & & \multicolumn{2}{|c|}{$5^{\circ} \mathrm{C}$} & \multicolumn{2}{|c|}{$15 / 4^{\circ} \mathrm{C}$} & \multicolumn{2}{|c|}{$20 / 7^{\circ} \mathrm{C}$} & \multicolumn{2}{|c|}{$25 / 10^{\circ} \mathrm{C}$} \\
\hline & & $\mathrm{L}$ & $\mathrm{O}$ & $\mathrm{L}$ & $\mathrm{O}$ & $\mathrm{L}$ & $\mathrm{O}$ & $\mathrm{L}$ & $\mathrm{O}$ \\
\hline \multirow{2}{*}{ E1 } & $\mathrm{L}$ & $55 \pm 1^{\mathrm{Cb}}$ & $62 \pm 1^{\mathrm{Cc}}$ & $97 \pm 2^{\mathrm{Cd}}$ & $100 \pm 0^{\text {Dd }}$ & $57 \pm 1^{\mathrm{Cb}}$ & $92 \pm 1^{\text {Dd }}$ & $0 \pm 0^{\mathrm{Aa}}$ & $0 \pm 0^{\mathrm{Aa}}$ \\
\hline & $\mathrm{O}$ & $42 \pm 1^{\mathrm{Cb}}$ & $95 \pm 2^{\mathrm{Dc}}$ & $99 \pm 1^{\mathrm{Cd}}$ & $100 \pm 0^{\text {Dd }}$ & $62 \pm 1^{\mathrm{Db}}$ & $90 \pm 2^{\mathrm{Dc}}$ & $0 \pm 0^{\mathrm{Aa}}$ & $1 \pm 0^{\mathrm{Aa}}$ \\
\hline \multirow{2}{*}{ E2 } & $\mathrm{L}$ & $0 \pm 0^{\mathrm{Aa}}$ & $32 \pm 1^{\mathrm{Bc}}$ & $97 \pm 1^{\mathrm{Cd}}$ & $99 \pm 1^{\text {Dd }}$ & $6 \pm 0^{\mathrm{Bb}}$ & $35 \pm 1^{\mathrm{Cc}}$ & $0 \pm 0^{\mathrm{Aa}}$ & $0 \pm 0^{\mathrm{Aa}}$ \\
\hline & $\mathrm{O}$ & $14 \pm 1^{\mathrm{Bb}}$ & $99 \pm 1^{\mathrm{Dd}}$ & $98 \pm 1^{\mathrm{Cd}}$ & $100 \pm 0^{\mathrm{Dd}}$ & $6 \pm 0^{\mathrm{Bab}}$ & $34 \pm 0^{\mathrm{Cc}}$ & $0 \pm 0^{\mathrm{Aa}}$ & $1 \pm 0^{\mathrm{Aa}}$ \\
\hline \multirow{2}{*}{ E3 } & $\mathrm{L}$ & $0 \pm 0^{\mathrm{Aa}}$ & $0 \pm 0^{\mathrm{Aa}}$ & $0 \pm 0^{\mathrm{Aa}}$ & $0 \pm 0^{\mathrm{Aa}}$ & $0 \pm 0^{\text {Aa }}$ & $0 \pm 0^{\mathrm{Aa}}$ & $0 \pm 0^{\mathrm{Aa}}$ & $0 \pm 0^{\mathrm{Aa}}$ \\
\hline & $\mathrm{O}$ & $0 \pm 0^{\mathrm{Aa}}$ & $0 \pm 0^{\mathrm{Aa}}$ & $0 \pm 0^{\mathrm{Aa}}$ & $0 \pm 0^{\mathrm{Aa}}$ & $0 \pm 0^{\mathrm{Aa}}$ & $0 \pm 0^{\mathrm{Aa}}$ & $0 \pm 0^{\mathrm{Aa}}$ & $0 \pm 0^{\mathrm{Aa}}$ \\
\hline
\end{tabular}


Para las semillas de $S$. ramburei, en dos de los ensayos de estratificación tipo "move-along" (E3 y E4), se alcanzó el $100 \%$ de germinación a $15 / 4{ }^{\circ} \mathrm{C}$ y $20 / 7{ }^{\circ} \mathrm{C}$ en ambas condiciones de iluminación. Asimismo, en las semillas estratificadas a $28 / 14{ }^{\circ} \mathrm{C}$ durante 24 semanas se alcanzaron porcentajes de germinación $\geq 70 \%$ en todas las temperaturas de incubación (cuadro 4). En el ensayo control durante 28 semanas de incubación, el porcentaje de germinación más alto $(8 \%)$ se alcanzó a $15 / 4^{\circ} \mathrm{C}$ en luz.

Efectos del $\mathrm{GA}_{3}$ sobre la capacidad germinativa. En $S$. paui, tras cuatro semanas a $20 / 7{ }^{\circ} \mathrm{C}$ en presencia de $\mathrm{GA}_{3}$ la germinación fue del $8 \%$ y tras cuatro semanas más se llegó al $77 \%$. Este dato contrasta con el $4 \%$ obtenido tras ocho semanas a $20 / 7{ }^{\circ} \mathrm{C}$ en semillas no tratadas con $\mathrm{GA}_{3}$. El GA $\mathrm{G}_{3}$ no estimuló la germinación de las semillas de S. ramburei, que fue nula al final del ensayo (datos no mostrados)

Inducción de latencia por altas temperaturas en S. paui. Cuando las semillas que habían salido de la latencia tras ocho semanas de estratificación fría a $5{ }^{\circ} \mathrm{C}$ fueron expuestas a $25 / 10^{\circ} \mathrm{C}$ durante cuatro semanas, entraron de nuevo en un estado de latencia. Para romper esta latencia secundaria se requirieron otras ocho semanas de estratificación fría a $5{ }^{\circ} \mathrm{C}$ (cuadro 5 ).
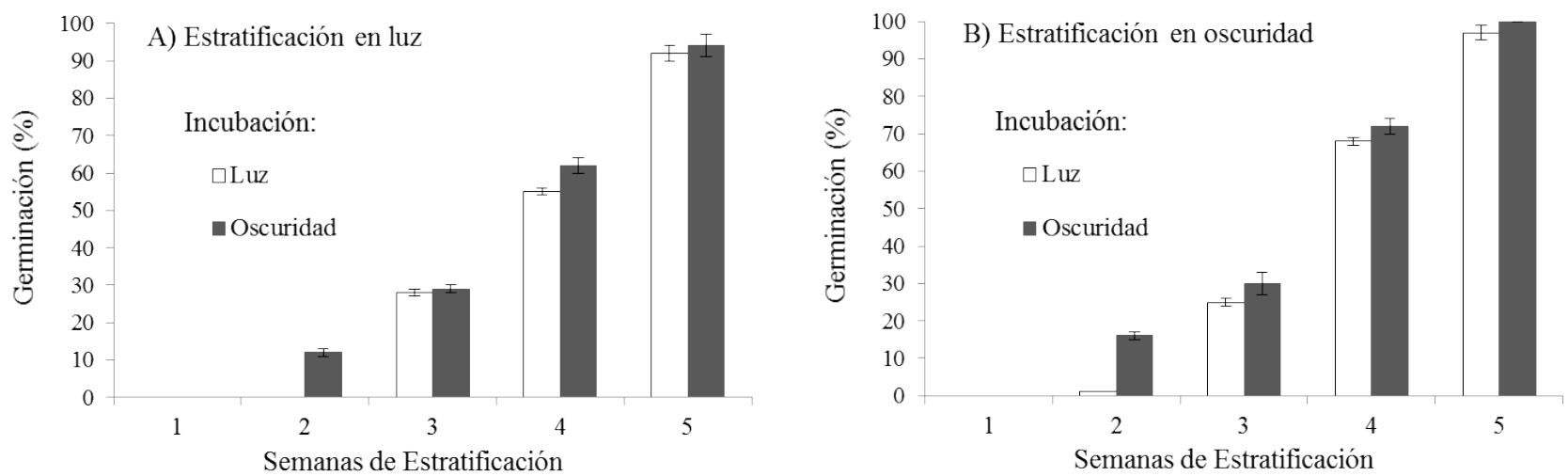

Figura 1. Porcentaje de germinación de $S$. paui (media $\pm \mathrm{SE}, \mathrm{n}=4$ ) obtenido tras la estratificación fría a $5^{\circ} \mathrm{C}$ en luz $(\mathrm{A}) \mathrm{u}$ oscuridad (B), durante 1, 2, 3, 4 y 5 semanas y posteriormente incubadas cuatro semanas a $15 / 4{ }^{\circ} \mathrm{C}$ en ambas condiciones de iluminación.

Germination percentage recorded in $S$. paui (mean $\pm \mathrm{SE}, \mathrm{n}=4$ ) after cold stratification at $5{ }^{\circ} \mathrm{C}$ in light $(\mathrm{A})$ or darkness $(\mathrm{B})$ during $1,2,3$, 4 , and 5 weeks, followed by a $15 / 4{ }^{\circ} \mathrm{C}$ incubation at light and darkness, respectively, during four weeks.

Cuadro 4. Porcentaje de germinación (media $\pm \mathrm{SE}, \mathrm{n}=4$ ) en semillas de $S$. ramburei sometidas a diferentes tratamientos de estratificación en luz, seguida de cuatro semanas de incubación a $5{ }^{\circ} \mathrm{C}, 15 / 4{ }^{\circ} \mathrm{C}$ y $20 / 7{ }^{\circ} \mathrm{C}$, tanto en luz (L) como en oscuridad (O). Los tratamientos de estratificación son los indicados en el cuadro 1. Las letras mayúsculas en columnas y minúsculas en filas denotan diferencias significativas $(P<0,05)$.

Germination percentage (mean $\pm \mathrm{SE} ; \mathrm{n}=4$ ) in $S$. ramburei seeds submitted to different stratification treatments in light, followed by four weeks of incubation at $5{ }^{\circ} \mathrm{C}, 15 / 4{ }^{\circ} \mathrm{C}$ and $20 / 7^{\circ} \mathrm{C}$, both in light (L) and darkness (O). Stratification treatments are the same as those indicated in Table 1. Different uppercase letters in columns and lowercase letters in rows denote significant differences $(P<0.05)$.

\begin{tabular}{|c|c|c|c|c|c|c|}
\hline \multirow{3}{*}{$\begin{array}{l}\text { Tratamiento de } \\
\text { estratificación }\end{array}$} & \multicolumn{6}{|c|}{ Temperatura de incubación } \\
\hline & \multicolumn{2}{|c|}{$5^{\circ} \mathrm{C}$} & \multicolumn{2}{|c|}{$15 / 4{ }^{\circ} \mathrm{C}$} & \multicolumn{2}{|c|}{$20 / 7^{\circ} \mathrm{C}$} \\
\hline & $\mathrm{L}$ & $\mathrm{O}$ & $\mathrm{L}$ & $\mathrm{O}$ & $\mathrm{L}$ & $\mathrm{O}$ \\
\hline E1 & $2 \pm 0^{\mathrm{Aa}}$ & $2 \pm 1^{\mathrm{Aa}}$ & $4 \pm 1^{\text {Aab }}$ & $8 \pm 1^{\mathrm{ABb}}$ & $1 \pm 0^{\mathrm{Aa}}$ & $2 \pm 0^{\mathrm{Aa}}$ \\
\hline E2 & $0 \pm 0^{\mathrm{Aa}}$ & $0 \pm 0^{\mathrm{Aa}}$ & $2 \pm 0^{\mathrm{Aab}}$ & $4 \pm 1^{\mathrm{Ab}}$ & $0 \pm 0^{\mathrm{Aa}}$ & $2 \pm 0^{\mathrm{Aa}}$ \\
\hline E3 & $81 \pm 0^{\mathrm{Ca}}$ & $91 \pm 0^{\mathrm{Cab}}$ & $100 \pm 0^{\mathrm{Cb}}$ & $100 \pm 0^{\mathrm{Cb}}$ & $100 \pm 0^{\mathrm{cb}}$ & $100 \pm 0^{\mathrm{Cb}}$ \\
\hline E4 & $92 \pm 0^{\mathrm{Ca}}$ & $93 \pm 0^{\mathrm{Ca}}$ & $100 \pm 0^{\mathrm{Cb}}$ & $100 \pm 0^{\mathrm{Cb}}$ & $100 \pm 0^{\mathrm{Cb}}$ & $100 \pm 0^{\mathrm{Cb}}$ \\
\hline E5 & $3 \pm 0^{A a}$ & $3 \pm 0^{\mathrm{Aa}}$ & $4 \pm 0^{\text {Aa }}$ & $4 \pm 1^{\mathrm{Aa}}$ & $4 \pm 1^{\text {Aa }}$ & $3 \pm 0^{\mathrm{Aa}}$ \\
\hline E6 & $16 \pm 0^{\mathrm{Ba}}$ & $16 \pm 2^{\mathrm{Ba}}$ & $12 \pm 0^{\mathrm{Ba}}$ & $20 \pm 1^{\mathrm{Ba}}$ & $8 \pm 1^{\mathrm{Ba}}$ & $12 \pm 1^{\mathrm{Ba}}$ \\
\hline E7 & $71 \pm 0^{\mathrm{Ca}}$ & $90 \pm 0^{\mathrm{Cb}}$ & $100 \pm 0^{\mathrm{Cc}}$ & $100 \pm 0^{\mathrm{Cc}}$ & $84 \pm 0^{\mathrm{Cb}}$ & $100 \pm 0^{\mathrm{Cc}}$ \\
\hline E8 & $0 \pm 0^{\mathrm{Aa}}$ & $0 \pm 0^{\mathrm{Aa}}$ & $0 \pm 0^{\mathrm{Aa}}$ & $0 \pm 0^{A a}$ & $0 \pm 0^{\mathrm{Aa}}$ & $0 \pm 0^{\mathrm{Aa}}$ \\
\hline
\end{tabular}


Cuadro 5. Porcentaje de germinación (media $\pm \mathrm{SE}, \mathrm{n}=4$ ) en semillas de $S$. paui expuestas a diferentes tratamientos de estratificación en luz y luego incubadas durante cuatro semanas a $15 / 4{ }^{\circ} \mathrm{C}$ tanto en luz (L) como en oscuridad (O).

Percentage of germination (mean $\pm \mathrm{SE}, \mathrm{n}=4$ ) in $S$. paui seeds exposed to different stratification treatments in light and afterward incubated during four weeks at $15 / 4{ }^{\circ} \mathrm{C}$, both in light (L) and in darkness (O).

\begin{tabular}{lrr}
\hline Tratamiento de estratificación & $\mathrm{L}$ & $\mathrm{O}$ \\
\hline E1: 8 semanas $5{ }^{\circ} \mathrm{C}($ control) & $97 \pm 2$ & $100 \pm 0$ \\
E2: 8 semanas $5{ }^{\circ} \mathrm{C}+4$ semanas $25 / 10^{\circ} \mathrm{C}$ & $0 \pm 0$ & $0 \pm 0$ \\
E3: 8 semanas $5{ }^{\circ} \mathrm{C}+4$ semanas $25 / 10^{\circ} \mathrm{C}+4$ semanas $5{ }^{\circ} \mathrm{C}$ & $0 \pm 0$ & $0 \pm 0$ \\
E4: 8 semanas $5{ }^{\circ} \mathrm{C}+4$ semanas $25 / 10^{\circ} \mathrm{C}+8$ semanas $5{ }^{\circ} \mathrm{C}$ & $92 \pm 1$ & $98 \pm 2$ \\
\hline
\end{tabular}

Efecto de la estratificación fría sobre la emergencia de plántulas en semillas de $\mathrm{S}$. ramburei con radículas emergidas. En las semillas con radículas emergidas no expuestas a $5{ }^{\circ} \mathrm{C}$ y colocadas directamente a $20 / 7^{\circ} \mathrm{C}$ en luz, solo se obtuvo un $13 \%$ de emergencia del cotiledón al final de los 60 días (figura 2). En las semillas con radículas emergidas expuestas durante cuatro semanas a $5^{\circ} \mathrm{C}$ se obtuvo un 53 $\%$ de emergencia del cotiledón a los 60 días a $20 / 7^{\circ} \mathrm{C}$ luz, y cuando habían transcurrido 32 días a $20 / 7^{\circ} \mathrm{C}$ en luz (es decir un total de 60 días, $28+32$, desde el inicio del ensayo) el porcentaje de emergencia del cotiledón ya superaba el $40 \%$. El cotiledón de las semillas con radículas emergidas que se expusieron ocho semanas a $5{ }^{\circ} \mathrm{C}$ emergió en un $78 \%$ tras solo 15 días a $20 / 7^{\circ} \mathrm{C}$, un $96 \%$ a los 30 días y un $100 \%$ a los 37 días (figura 2 ).

Fenología de crecimiento del embrión y emergencia de radículas y plántulas. Las semillas de S. paui enterradas el 1 de septiembre de 2009, inician la emergencia de radículas en diciembre de 2009 , alcanzando un $99 \%$ a finales de enero de 2010. La emergencia de plántulas se inició con un desfase de medio mes desde la emergencia de las radículas. A principios de enero de 2010 pudo observarse una emergencia de plántulas del $28 \%$, que llegó a ser del $94 \%$ a mediados de febrero de 2010 (figura 3).

Los embriones de las semillas de $S$, ramburei utilizadas en el ensayo de fenología iniciado el 1 de septiembre de 2009 , que poseían un tamaño inicial de 1,60 mm, iniciaron su crecimiento con las temperaturas cálidas de septiembre y octubre, alcanzando los 2,07 $\mathrm{mm}$ en diciembre de 2009 (figura 4). Debido al crecimiento rápido del embrión con las temperaturas suaves del otoño, se pudieron observar las primeras germinaciones (emergencias de radículas) en noviembre de 2009 , con un $8 \%$ que rápidamente alcanzó el $82 \%$ a principios de diciembre y el $99 \%$ a finales de diciembre- principios de enero de 2010 (figura 4).

Sin embargo, la emergencia del cotiledón no se observó hasta que se superaron las temperaturas más frías de diciembre y enero. Las primeras emergencias se produjeron en el mes de febrero de 2010, con un $7 \%$. En el mes de marzo la emergencia alcanzó un $48 \%$, viéndose completada con un $97 \%$ en abril de 2010 (figura 4).
Cambios en el estado de latencia de semillas enterradas y rescatadas periódicamente. En S. paui las primeras semillas germinadas en bolsa no aparecen hasta el rescate efectuado el 1 de enero de 2010, tras haber experimentado un periodo considerable (unas seis semanas) de estratificación fría, aunque la mayoría de las semillas rescatadas el 1 de diciembre ya han salido de la latencia. En cambio, en $S$. ramburei se enconraron semillas germinadas ya en el rescate efectuado el 1 de noviembre de 2009, sin apenas estratificación fría previa, y germinaron más del $80 \%$ con las temperaturas del mes de noviembre (figura 5).

\section{DISCUSIÓN}

El estudio del tamaño de los embriones de las semillas recién dispersadas de $S$. paui indica que esta especie no posee embriones subdesarrollados, pues el tamaño inicial es de $1,59 \mathrm{~mm}$, igual que el tamaño de los embriones en las semillas germinadas. Por ello, se puede descartar para esta especie una latencia de tipo morfológica sensu Baskin y Baskin (1998). Por otra parte, en los ensayos de influencia de la temperatura de estratificación y condiciones de iluminación sobre la capacidad germinativa iniciados en septiembre de 2009, se puede observar como en las semillas que habían sido sometidas a los tratamientos de estratificación fría E1 y E2 (ocho y seis semanas efectivas de estratificación fría, respectivamente), se supera el $95 \%$ de germinación a $15 / 4{ }^{\circ} \mathrm{C}$ (cuadro 3). En el ensayo control a $15 / 4{ }^{\circ} \mathrm{C}$ oscuridad también se supera este porcentaje porque dicho ensayo implica seis semanas efectivas de estratificación fría a $4{ }^{\circ} \mathrm{C}$. Incluso con periodos de estratificación fría de cuatro semanas, llegan a germinar más del $50 \%$ de las semillas. La superación de la latencia fisiológica con una estratificación fría de pocas semanas y la capacidad del $\mathrm{GA}_{3}$ para promover la germinación, indican que las semillas de $S$. paui presentan una latencia fisiológica no profunda (Baskin y Baskin 1998).

Los ensayos de fenología de germinación, emergencia de plántulas y rotura de latencia realizados con semillas de S. paui confirman lo observado en las pruebas de laboratorio, y muestran cómo las semillas precisan de la llegada de las temperaturas frías, que empiezan a mediados 


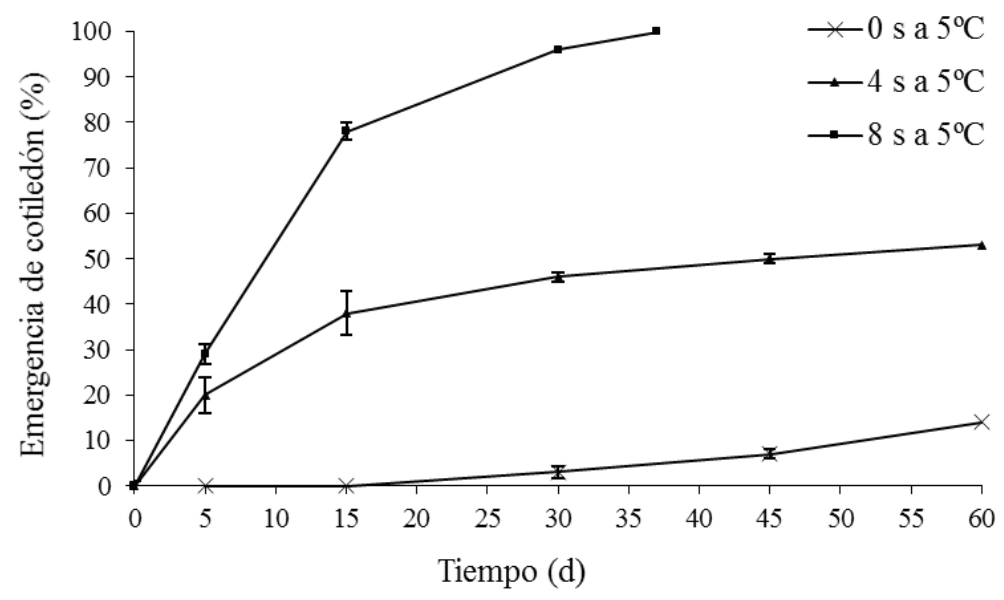

Figura 2. Porcentaje de emergencia del cotiledón en semillas con radículas emergidas de S. ramburei a $20 / 7^{\circ} \mathrm{C}$ en luz durante 60 días (d), después de 0,4 y 8 semanas (s) a $5{ }^{\circ} \mathrm{C}$.

Cotyledon-emergence percentage at $20 / 7{ }^{\circ} \mathrm{C}$ in light during 60 days, after 0,4 , and 8 weeks at $5{ }^{\circ} \mathrm{C}$, in $S$. ramburei seeds with radicle emerged.
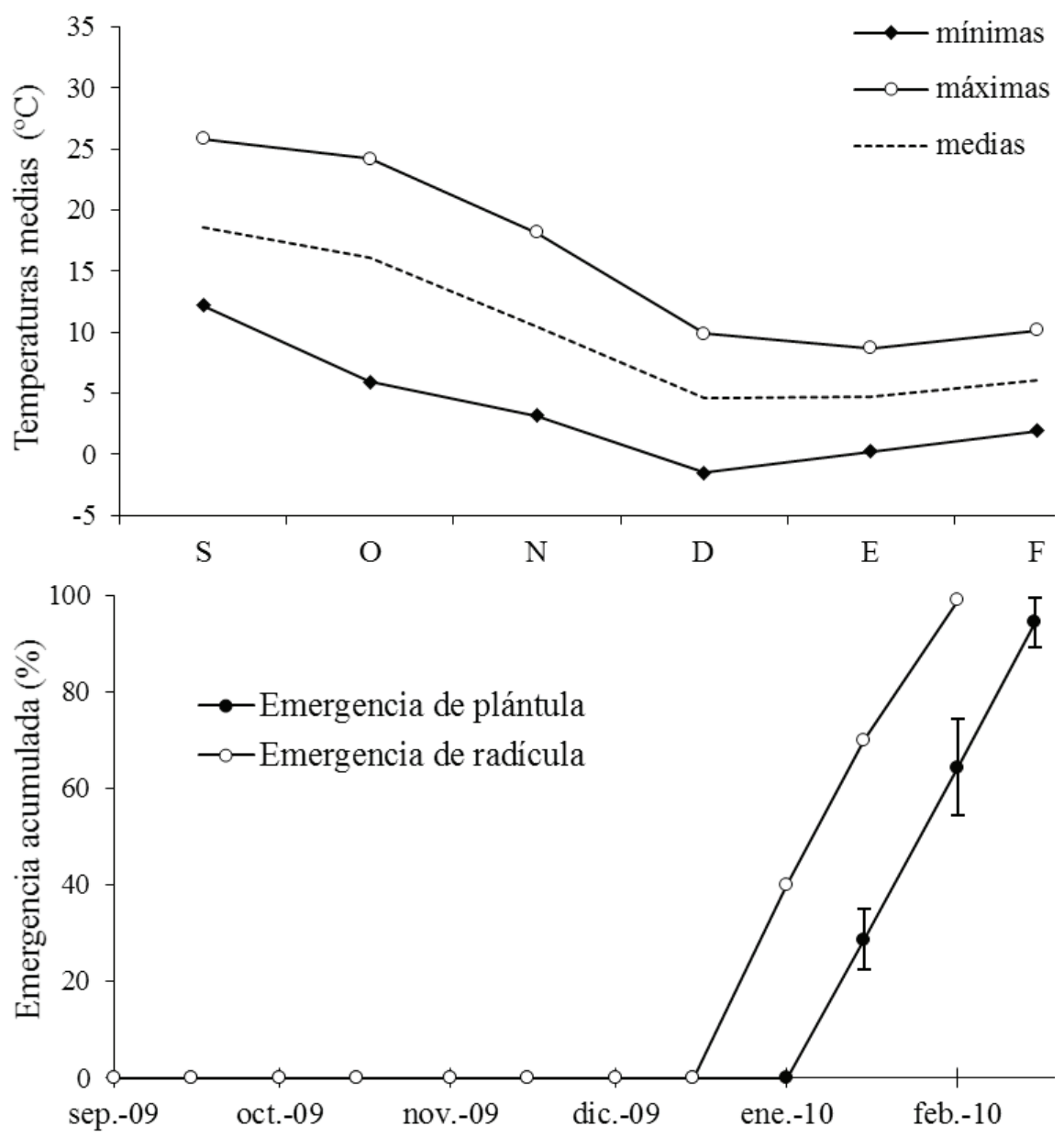

Figura 3. Fenología de la emergencia de radículas y plántulas (media $\pm \mathrm{SE}, \mathrm{n}=3$ ) en semillas de $S$. paui enterradas el 1 de septiembre de 2009. La gráfica superior muestra los cambios de temperatura (media de las máximas, media de las mínimas y temperaturas medias mensuales) producidos en el umbráculo a lo largo del experimento.

Phenology of germination and radicle and seedling emergence (mean $\pm \mathrm{SE}, \mathrm{n}=3$ ) in S. paui seeds buried on 1 September 2009. The upper figure shows changes in temperature (mean of maximum, minimum, and monthly values) recorded in the shade house throughout the experiment. 

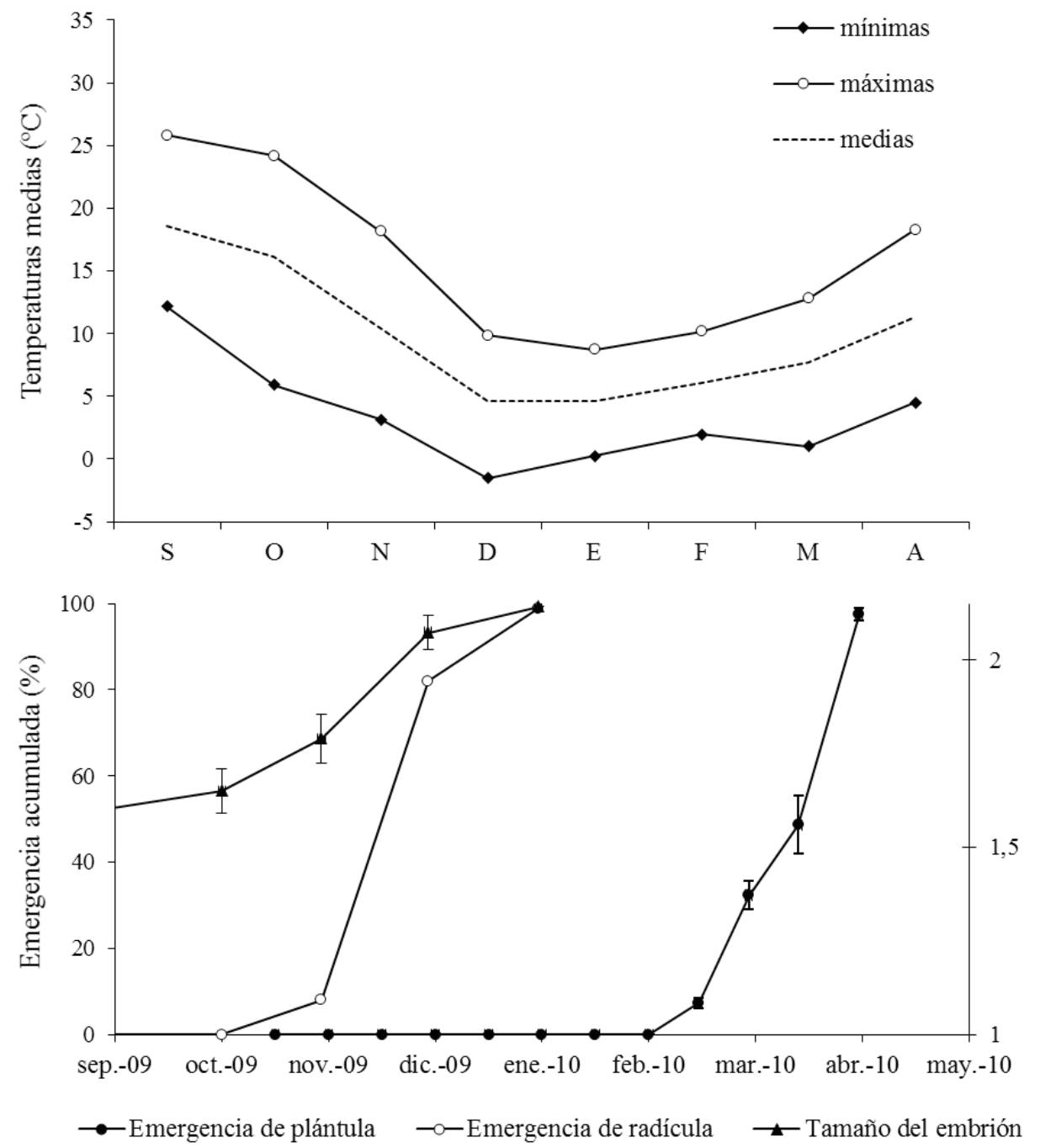

Figura 4. Fenología del crecimiento del embrión (media $\pm \mathrm{SE}$, si $\mathrm{SE}>0,1 ; \mathrm{n}=25$ ), emergencia de radículas y epicotilos (media $\pm \mathrm{SE}$, $\mathrm{n}=3$ ) en semillas de $S$. ramburei enterradas el 1 de septiembre de 2009. La gráfica superior muestra los cambios de temperatura (media de las máximas, media de las mínimas y temperaturas medias mensuales) producidos en el umbráculo a lo largo del experimento.

Phenology of embryo growth (mean $\pm \mathrm{SE}$, if $\mathrm{SE}>0.1 ; \mathrm{n}=25$ ) and of radicle and epicotyl emergence (mean $\pm \mathrm{SE}, \mathrm{n}=3$ ) in $S$. ramburei seeds buried on 1 September 2009. The upper figure shows changes in temperature (mean of maximum, minimum, and monthly values) recorded in the shade house throughout the experiment.

de noviembre, para conseguir superar la latencia y que se produzcan las primeras emergencias de radícula a mediados de diciembre, llegando a completarse en enero del siguiente año.

El comportamiento descrito para la emergencia de las radículas y la consecutiva e inmediata emergencia de las plántulas, implican que las plántulas de $S$. paui quedarían expuestas a las temperaturas de finales del invierno y principios de primavera en febrero y marzo. Esta característica de la latencia de $S$. paui podría ser considerada una ventaja adaptativa, que permitiría a sus plántulas establecerse una vez pasado el periodo más frío del invierno y antes que otras especies que germinan en primavera; esta emergencia temprana estaría relacionada con una mayor supervi- vencia al ser más competitivas las plántulas primeras en establecerse (Mercer et al. 2011).

Por otra parte, es conocido que la temperatura es uno de los factores que determinan el ciclo anual de latencias y la aparición de latencias secundarias (Hilhorst 1998). Así sucede en $S$. paui, donde se puede inducir una latencia secundaria en aquellas semillas que ya habían superado su latencia fisiológica primaria. En este sentido, las semillas de $S$. paui se comportan como muchas anuales de verano que salen de la latencia con el frío y son inducidas de nuevo al estado durmiente con el calor (Baskin y Baskin 1998) y como algunas vivaces, tales como Delphinium fissum Waldst. et Kit. (Herranz et al. 2010). En el caso de S. paui se da además la circunstancia de que la latencia secundaria 

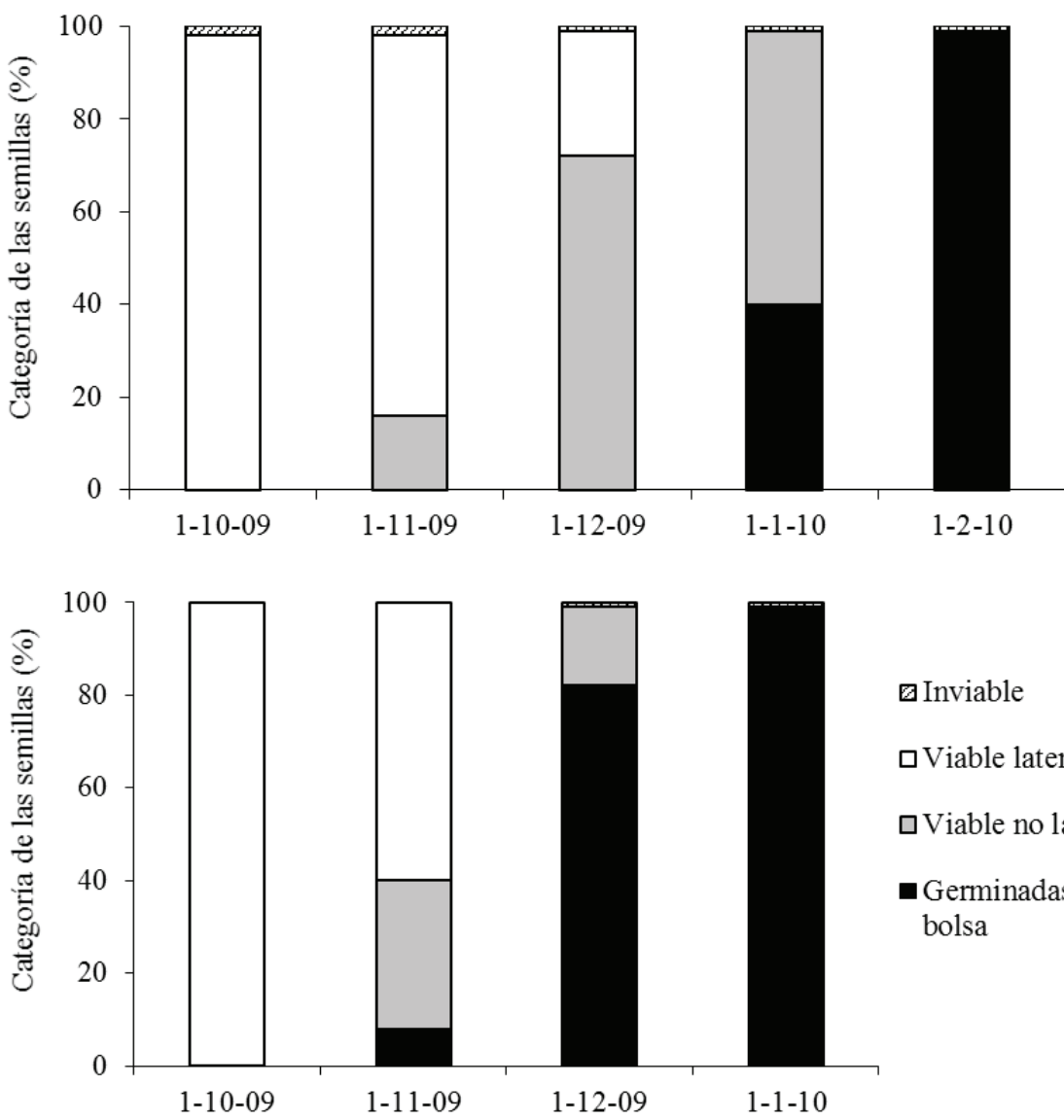

믐iable

$\square$ Viable latente

$\square$ Viable no latente

- Germinadas en la bolsa

Figura 5. Cambios en el estado de latencia de semillas enterradas. Porcentajes de semillas germinadas en la bolsa (radículas emergidas), viables latentes, viables no latentes e inviables de $S$. paui (A) y $S$. ramburei (B), enterradas el 1 de septiembre de 2009 y rescatadas con periodicidad mensual durante cinco y cuatro meses, respectivamente.

Changes in the dormancy stage of buried seeds. Percentage of seeds germinated (radicle emerged) within the bag, as well as dormant viable, non-dormant viable and unviable seeds of S. paui (A) and S. ramburei (B), buried on 1 September 2009 and exhumed monthly during a five- or fourmonth period, respectively.

inducida por altas temperaturas requiere un mayor periodo de estratificación fría para ser superada que la latencia primaria. Este mecanismo de inducción de latencia puede ser útil para evitar que las semillas no germinadas en la primera primavera post dispersión puedan salir de la latencia tras cortos periodos fríos otoñales $\mathrm{y}$, por tanto, germinar fuera de su ciclo fenológico habitual (Hilhorst 1998).

Los resultados obtenidos para $S$. paui, que indican la existencia de latencia fisiológica no profunda, confirman la hipótesis de partida. La necesidad de un periodo de estratificación fría mayor o igual a cinco semanas para la rotura de la latencia es un mecanismo eficaz para evitar la emergencia otoñal de plántulas y la exposición de estas al periodo invernal más frío.

En $S$. ramburei, la presencia de embriones claramente subdesarrollados en el momento de la dispersión de las semillas y la necesidad de largos periodos de estratificación cálida para poder emerger las radículas, indican que posee una latencia morfofisiológica (Baskin y Baskin 1998).
A pesar de los diferentes resultados obtenidos en las pruebas de laboratorio y las que se desarrollan en el umbráculo, ambos coinciden en que se precisan varios meses de estratificación cálida para que las semillas germinen, existiendo un retraso entre la emergencia de la radícula (germinación) y la emergencia del cotiledón. Para que se produzca la emergencia del cotiledón, las semillas con radículas emergidas han de ser expuestas a un periodo de estratificación fría, que bajo condiciones naturales se produce durante los meses de enero y febrero y en laboratorio se acelera tras ocho semanas a $5{ }^{\circ} \mathrm{C}$.

Teniendo en consideración lo anteriormente descrito, se puede concluir que las semillas de $S$. ramburei poseen una latencia morfofisiológica simple y profunda del epicotilo, en la que las radículas emergen en otoño y la emergencia del cotiledón se retrasa hasta el final del invierno, principio de la primavera. Esta secuencia ya fue descrita para diversas especies con latencia profunda simple del epicotilo, entre otras la ranunculácea Hepatica 
acutiloba DC. (Baskin y Baskin 1985b), la aristoloquiácea Hexastylis heterophylla (Ashe) Small (Adams et al. 2003), así como para las liliáceas Erythronium japonicum Decne. (Kondo et al. 2002) y Gagea lutea (Kondo et al. 2004). Dentro del género Scilla, dicha secuencia ha sido descrita anteriormente para S. bifolia (Vandelook y Van Assche 2008), si bien los autores, aun reconociendo el retraso de la emergencia del cotiledón (i.e. tallo), no lo consideran latencia del epicotilo, sino un simple retraso en el crecimiento debido a las temperaturas frías invernales.

Esta latencia profunda simple del epicotilo permitiría a S. ramburei que sus plántulas no se vieran sometidas a las heladas invernales o a la posible acción de predadores en la estación fría, permitiendo, sin embargo, que sus radículas emergidas en el otoño siguieran desarrollándose bajo el sustrato durante los meses invernales, produciéndose así un desarrollo más vigoroso de las plántulas cuando estas emerjan al finalizar el invierno y llegar la primavera. En esta especie, la exposición a las bajas temperaturas (5 ${ }^{\circ} \mathrm{C}$ ) al final del periodo de rotura de latencia no indujo una nueva latencia, al contrario de lo que sucede en Narcissus hispanicus Gouan, que también posee semillas con latencia profunda simple del epicotilo (Copete et al. 2011).

$\mathrm{Al}$ abordar el tratamiento del género Scilla, Almeida Da Silva y Crespi (2013) han agrupado los dos taxones objeto de este trabajo bajo la denominación de S. verna Huds. subsp. ramburii (Boiss.) K. Richt. Sin embargo, las diferencias entre ambos taxones en requerimientos ambientales para la rotura de latencia y germinación, puestas de manifiesto en este estudio, reflejan la existencia de características fisiológicas diferenciadas, que unidas a los caracteres morfológicos diferenciadores con rango específico indicados por Navarro (2009), apoyan el mantenimiento de ambos taxones como especies diferentes.

\section{CONCLUSIONES}

Las semillas de $S$. paui tienen latencia fisiológica no profunda: embriones desarrollados al dispersar y requieren un periodo corto de estratificación fría para la rotura de la latencia.

Las semillas de $S$. ramburei tienen latencia morfofisiológica simple y profunda del epicotilo: embriones subdesarrollados al dispersar, emergencia de radículas tras temperaturas moderadamente cálidas y de plántulas tras un periodo frío.

Los conocimientos sobre ecología germinativa aportan datos relevantes a ser tenidos en cuenta en los estudios de sistemática.

\section{AGRADECIMIENTOS}

A los revisores del artículo por las sugerencias realizadas, que han permitido facilitar su comprensión y que mejore apreciablemente la calidad del mismo.

\section{REFERENCIAS}

Adams CA, JM Baskin, CC Baskin. 2003. Epicotyl dormancy in the mesic woodland herb Hexastylis heterophylla (Aristolochiaceae). Journal of the Torrey Botanical Society 130: 11-15.

Almeida Da Silva RM, AL Crespi. 2013. Scilla L. In Castroviejo S, C Aedo, M Laínz, F Muñoz-Garmendia, G Nieto-Feliner, J Paiva, C Benedí eds. Flora Iberica XII. Real Jardín Botánico, Madrid, España. CSIC. p. 234-288.

Baskin CC, JM Baskin. 1998. Seeds: ecology, biogeography, and evolution of dormancy and germination. San Diego, California. Academic Press. 666 p.

Baskin CC, CT Chien, SY Chen, JM Baskin. 2008. Germination of Viburnum odoratissimum seeds: a new level of morphophysiological dormancy. Seed Science Research 18: 179184.

Baskin CC, SE Meyer, JM Baskin. 1995. Two types of morphophysiological dormancy in seeds of two genera (Osmorhiza and Erythronium) with an arcto-tertiary distribution pattern. American Journal of Botany 82: 293-298.

Baskin JM, CC Baskin. 1985a. Seed germination ecophysiology of the woodland spring geophyte Erythronium albidum. Botanical Gazette 146: 130-136.

Baskin JM, CC Baskin. 1985b. Epicotyl dormancy in seeds of Cimifuga racemosa and Hepatica acutiloba. Bulletin of the Torrey Botanical Club 112: 253-257.

Baskin JM, CC Baskin. 2004. A classification system for seed dormancy. Seed Science Research 14: 1-16.

Cerabolini B, R De Andrés, RM Ceriani, S Pierce, B Raimondi. 2004. Seed germination and conservation of endangered species from the Italian Alps: Physoplexis comosa and Primula glaucescens. Biological Conservation 117: 351-356.

Copete E, JM Herranz, P Ferrandis, CC Baskin, JM Baskin. 2011. Physiology, morphology and phenology of seed dormancybreak and germination in the endemic Iberian species Narcissus hispanicus (Amaryllidaceae). Annals of Botany 107: 1003-1016.

DOCM (Diario Oficial de Castilla-La Mancha, ES). 1998. Decreto 33/1998, de 05-05-98, por el que se crea el Catálogo Regional de Especies Amenazadas de Castilla-La Mancha. Diario Oficial de Castilla-La Mancha 22: 3391-3398.

Herranz JM, P Ferrandis, E Martínez-Duro. 2010. Seed germination ecology of the threatened endemic Iberian Delphinium fissum subsp. sordidum (Ranunculaceae). Plant Ecology 211: 89-106.

Herranz JM, JM Herrero, MA Copete, JA Monreal, P Ferrandis. 2011. Evaluación de riesgos de extinción y prioridades de conservación de la flora silvestre y vegetación de CastillaLa Mancha. In Bermejo JE, JM Herranz eds. Protección de la diversidad vegetal y de los recursos fitogenéticos en Castilla-La Mancha. Albacete, España. Instituto de Estudios Albacetenses "Don Juan Manuel”. p. 103-128.

Hilhorst HWM. 1998. The regulation of secondary dormancy: the membrane hypothesis revisited. Seed Science Research 8: 77-90.

Kondo T, N Okubo, T Miura, K Honda, Y Ishikawa. 2002. Ecophysiology of seed germination in Erythronium japonicum (Liliaceae) with underdeveloped embryos. American Journal of Botany 89(11): 1779-1784.

Kondo T, T Miura, N Okubo, M Shimada, CC Baskin, JM Baskin. 
2004. Ecophysiology of deep simple epicotyl morphophysiological dormancy in seeds of Gagea lutea (Liliaceae). Seed Science Research 14: 371-378.

Kondo T, C Sato, JM Baskin, CC Baskin. 2006. Post-dispersal embryo development, germination phenology, and seed dormancy in Cardiocrinum cordatum var. glehnii (Liliaceae s. Str.), a perennial herb of the broadleaved deciduous forest in Japan. American Journal of Botany 93(6): 849-859.

Marques I, D Draper. 2012. Seed germination and longevity of autumn-flowering and autumn-seed producing Mediterranean geophytes. Seed Science Research 22(4): 299-309.

Mercer KL, MH Alexander, AA Snow. 2011. Selection on seedling emergence timing and size an annual plant, Helianthus annuus (common sunflower, Asteraceae). American Journal of Botany 98(6): 975-985.

Navarro FB. 2009. Scilla L. In Blanca G, B Cabezudo, M Cueto, C Fernández-López, C Morales-Torres eds. Flora Vascular de Andalucía Oriental. Sevilla, España. Junta de Andalucía. p. $154-156$.

Phartyal SS, T Kondo, Y Hoshino, CC Baskin, JM Baskin. 2009. Morphological dormancy in seeds of the autumn-germinating shrub Lonicera caerulea var. emphyllocalyx (Caprifoliaceae). Plant Species Biology 24: 20-26.

Vandelook F, JA Van Assche. 2008. Temperature Requirements for Seed Germination and Seedling Emergence of three Monocotyledonous Temperate Forest Spring Geophytes. Annals of Botany 102: 865-875.

Vandelook F, N Bolle, JA VanAsche. 2009. Morphological and physiological dormancy in seeds of Aegopodium podagraria (Apiaceae) broken successively during cold stratification. Seed Science Research 19: 115-123.

Washitani I, M Masuda. 1990. A comparative study of the germination characteristics of seeds from a moist tall grassland community. Functional Ecology 4: 543-557. 
